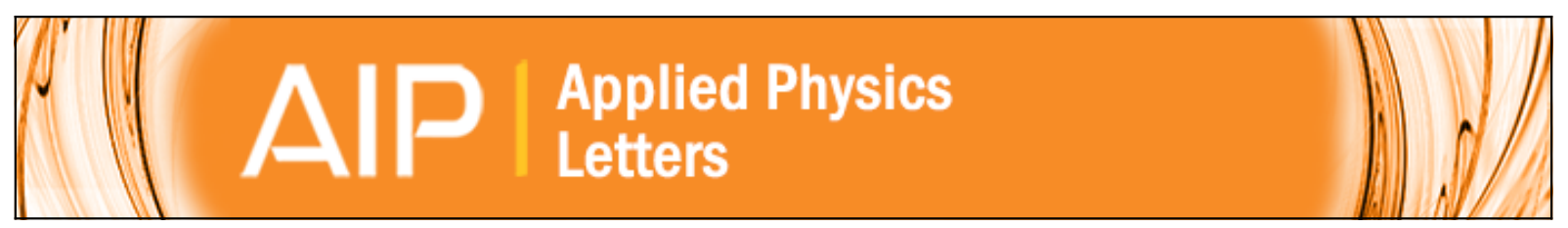

\title{
Observations of a mode transition in a hydrogen hollow cathode discharge using phase resolved optical emission spectroscopy
}

Sam Dixon, Christine Charles, James Dedrick, Timo Gans, Deborah O'Connell, and Rod Boswell

Citation: Applied Physics Letters 105, 014104 (2014); doi: 10.1063/1.4889916

View online: http://dx.doi.org/10.1063/1.4889916

View Table of Contents: http://scitation.aip.org/content/aip/journal/apl/105/1?ver=pdfcov

Published by the AIP Publishing

\section{Articles you may be interested in}

The plasma properties and electron emission characteristics of near-zero differential resistance of hollow cathode-based plasma contactors with a discharge chamber

Phys. Plasmas 21, 083506 (2014); 10.1063/1.4892953

Observation of radio frequency ring-shaped hollow cathode discharge plasma with $\mathrm{MgO}$ and $\mathrm{Al}$ electrodes for plasma processing

J. Vac. Sci. Technol. A 32, 031304 (2014); 10.1116/1.4871467

Criteria of radio-frequency ring-shaped hollow cathode discharge using $\mathrm{H} 2$ and $\mathrm{Ar}$ gases for plasma processing J. Appl. Phys. 113, 033302 (2013); 10.1063/1.4776220

Development of an atmospheric-pressure homogeneous and cold $\mathrm{Ar}$ / O 2 plasma source operating in glow discharge

Phys. Plasmas 17, 063506 (2010); 10.1063/1.3447877

Doppler spectroscopy of hydrogen Balmer lines in a hollow cathode glow discharge in ammonia and argonammonia mixture

Phys. Plasmas 15, 113501 (2008); 10.1063/1.2999535

\section{A|P| $\begin{aligned} & \text { Journal of } \\ & \text { Applied Physics }\end{aligned}$}

Journal of Applied Physics is pleased to announce André Anders as its new Editor-in-Chief 


\title{
Observations of a mode transition in a hydrogen hollow cathode discharge using phase resolved optical emission spectroscopy
}

\author{
Sam Dixon, ${ }^{1, a)}$ Christine Charles, ${ }^{1}$ James Dedrick, ${ }^{1}$ Timo Gans, ${ }^{2}$ Deborah O'Connell, ${ }^{2}$ \\ and Rod Boswell ${ }^{1}$ \\ ${ }^{1}$ Space Plasma, Power and Propulsion Laboratory, Research School of Physics and Engineering, \\ The Australian National University, Canberra ACT 0200, Australia \\ ${ }^{2}$ Department of Physics, York Plasma Institute, University of York, Heslington, York YO10 5DD, \\ United Kingdom
}

(Received 9 May 2014; accepted 30 June 2014; published online 9 July 2014)

\begin{abstract}
Two distinct operational modes are observed in a radio frequency (rf) low pressure hydrogen hollow cathode discharge. The mode transition is characterised by a change in total light emission and differing expansion structures. An intensified CCD camera is used to make phase resolved images of Balmer $\alpha$ emission from the discharge. The low emission mode is consistent with a typical $\gamma$ discharge, and appears to be driven by secondary electrons ejected from the cathode surface. The bright mode displays characteristics common to an inductive discharge, including increased optical emission, power factor, and temperature of the $\mathrm{H}_{2}$ gas. The bright mode precipitates the formation of a stationary shock in the expansion, observed as a dark region adjacent to the source-chamber interface. (C) 2014 AIP Publishing LLC. [http://dx.doi.org/10.1063/1.4889916]
\end{abstract}

Arrayed hollow cathode systems are useful for plasma processing applications due to their high plasma density and scalable geometry. ${ }^{1}$ Hydrogen is often used for surface processing applications such as film or crystal growth ${ }^{2,3}$ or the deposition of hydrogenated silicon. "The "Pocket Rocket" source $^{5}$ provides a useful analogue of a single hollow cathode that might appear in such an array. ${ }^{6}$ To optimise the operation of such a source, a detailed understanding of phase resolved electron dynamics is necessary.

Intensified Charge Coupled Devices (ICCDs) are an efficient tool for time resolved two dimensional imaging of a plasma. ${ }^{7}$ Through the use of a gated intensifier they can provide resolution well below the period of an $\mathrm{rf}$ cycle. ${ }^{8} \mathrm{By}$ virtue of this, they are capable of probing nanosecond scale phenomena within a discharge. Such measurements can provide insight into the mechanisms that drive discharge behaviour. ${ }^{9}$ Additionally, the non-invasive nature of the diagnostic is essential to avoid perturbing the system under measurement in experiments, such as Pocket Rocket, that are constrained by small length scales.

Much of the work using ICCD cameras to investigate micro to millimetre scale discharges has been performed at atmospheric pressure and with microsecond resolution. ${ }^{10}$ However, since wafer processing normally occurs in a vacuum environment, the experiments presented in this Letter are performed below atmosphere, at pressures of approximately 1 Torr. While operating in $\mathrm{H}_{2}$, the light emitted by the Pocket Rocket discharge is dominated by the Balmer $\alpha$ line.

The Pocket Rocket device, shown in Figure 1, is a millimetre scale discharge ignited inside a cylindrical alumina sleeve $20 \mathrm{~mm}$ long. ${ }^{5,11,12}$ The sleeve has a copper electrode $5 \mathrm{~mm}$ wide encircling its centre, forming a hollow cathode. The electrode is driven at $12.5 \mathrm{MHz}(80 \mathrm{~ns}$ period) via a pi

a)sam.dixon@anu.edu.au matching network. ${ }^{12}$ At either end of the alumina sleeve grounded aluminium electrodes $3 \mathrm{~mm}$ wide connect to the plenum and expansion tube.

During operation, $\mathrm{H}_{2}$ flows into the plenum where pressure is measured with a manometer. The gas passes through the alumina sleeve where breakdown occurs before passing into the low pressure region in the expansion tube. The expansion tube is a glass cylinder $5 \mathrm{~cm}$ in diameter and $10 \mathrm{~cm}$ in length. The system attaches to a six way cross chamber that is in turn connected to a rotary pump. The base pressure in the system is $1 \mathrm{mTorr}$, while operating pressures are between 0.4 and 2.2 Torr. The low gas conductance between the plenum and the expansion tube means there is a pressure gradient through the alumina sleeve. Typically, the pressure in the plenum is around 2.5 times greater than that in the expansion



FIG. 1. Schematic diagram of the Pocket Rocket system and the associated power delivery and data acquisition system. 
chamber. Measurements of the pressure in the expansion tube using a translatable Pitot tube exhibit no significant pressure variation in the downstream region.

Optical access is available from the rear, looking through the plenum down the axis of the hollow cathode, and from the side, looking at the plasma plume in the glass expansion tube. The orientation of the ICCD for the rear position is shown in Figure 1, for the side view the ICCD is perpendicular to the expansion tube. Between the ICCD and the Pocket Rocket source a lens is used to focus the image as well as magnify it such that spatial resolution is maximised.

For each $80 \mathrm{~ns}$ rf cycle, the ICCD acquires 40 images at $2 \mathrm{~ns}$ intervals. To achieve this temporal resolution, the voltage applied to the powered electrode is phase locked to the trigger signal for the ICCD camera. Both a high voltage (HV) probe and an Impedans Octiv IV probe are placed between the matchbox and driven electrode (Figure 1). As such, the actual voltage on the driven electrode at the time of each image's acquisition is measured simultaneously with the impedance and phase of the system during operation.

Two distinct operational modes are observed, dependent on the pressure power product of the system with the plasma ignited. These modes are named after their appearances in the expansion chamber. Using the pressure in the plenum as a guide, below 11 Torr $\mathrm{W}$, the plasma is said to operate in the "diffuse" mode. Above this threshold, the discharge operates in the "bright" mode, and total optical emission is an order of magnitude more intense. There are significant changes to the emission structure within an rf cycle for the two modes. Both modes display a single dominant plasma extrusion into the expansion tube once per rf cycle. The speed of this extrusion in both modes is measured to be approximately $2 \times 10^{6} \mathrm{~ms}^{-1}$, similar to the speed of an ionising electron. It is suggested that the extrusions of plasma in both modes are driven by bursts of electrons propagating down the expansion tube. The timing between the maximum penetration of each mode varies by approximately half an $\mathrm{rf}$ cycle as shown in Figure 2.

Figures 2(a) and 2(b), respectively, show examples of the diffuse and bright modes from the side view at the time the plasma penetrates furthest into the expansion tube. The insets in each of the two images are taken from the HV probe signal and show the position within the rf cycle corresponding to the image. The intensity of each of the two images is displayed on the same logarithmic scale to aid visualisation of the key components of the emission structure.

Panel (c) shows variation in emission intensity for both modes over the course of an rf cycle. Emission is spatially integrated over the region of interest (ROI) shown as a black box in panel (a), measuring $5 \times 5 \mathrm{~mm}$. Panel (d) shows the intensity over an rf cycle from the rear view for an ROI in the centre of the hollow cathode, with a size of $2 \times 2 \mathrm{~mm}$. Since the intensity of the emission varies strongly between the two modes the spatially integrated intensity for each ROI is normalised to its own maximum value to facilitate comparison of the structure between the two modes.

Time averaged ro-vibrational spectroscopy of the $v^{\prime}=2$ $\rightarrow v^{\prime \prime}=2$ Fulcher $\alpha$ band yields translational temperatures of the $\mathrm{H}_{2}$ gas during operation. ${ }^{13}$ The gas temperature is measured to be between 310 and $375 \mathrm{~K}$ for the diffuse mode as power varies between 22.5 and $30 \mathrm{~W}$. The transition to the bright mode increases the temperature to between 450 and $550 \mathrm{~K}$ for powers of $32.5-40 \mathrm{~W}$. Similar results were obtained by Abdel-Rahman et al. observing a capacitive to inductive (E-H) transition in a planar hydrogen reactor. ${ }^{14}$

Figure 3 shows time averaged measurements of the plasma's resistance as well as power deposited in the discharge with respect to plenum pressure. The IV and HV probes provide simultaneous measurements of voltage, current, phase, and complex impedance. The resistance and reactance are directly taken from the impedance. The deposited power is defined by the product of the voltage, current, and power
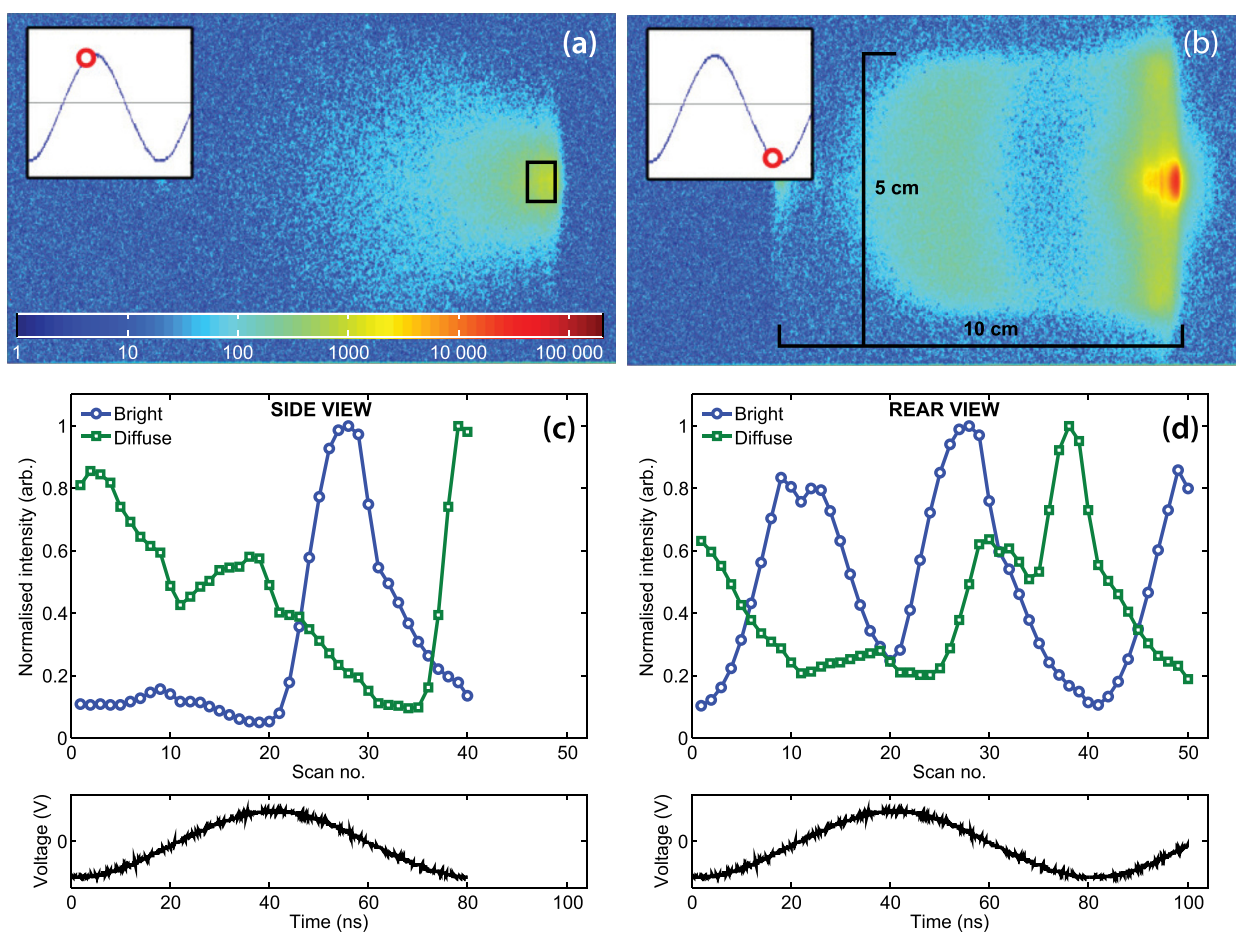

FIG. 2. Panels (a) and (b) show the Balmer $\alpha$ emission intensity of the expanding plasma in the diffuse and bright modes, respectively, as viewed from the side. The insets in the top left of (a) and (b) show the position within the rf cycle when the image was acquired. The black box in (a) defines the ROI analysed in panel (c), which shows the normalised integrated intensity count for the ROI in both modes. Panel (d) shows the normalised integrated intensity count for a central ROI for the rear view, looking directly into the hollow cathode source. 


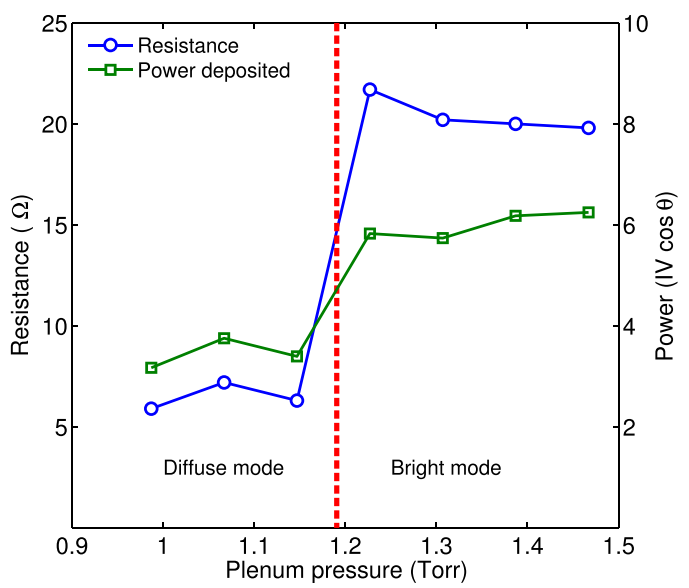

FIG. 3. Variation of the plasma resistance and deposited power with changing pressure. The dashed red line serves as a visual cue to distinguish the two modes of operation.

factor, $\cos \theta$. Deposited power increases by a factor of two during the transition from the diffuse to the bright mode and the system resistance by a factor of four. For the diffuse mode shown in Figure 3, voltage, current, and phase are $275 \mathrm{~V}, 0.72 \mathrm{~A}$, and $89.0^{\circ}$, respectively. The bright mode is $190 \mathrm{~V}, 0.53 \mathrm{~A}$, and $86.4^{\circ}$. Although both current and voltage are lower in the bright mode the marked increase in power factor implies an increase in total power deposited in the system.

As shown in panel (d) of Figure 2, the diffuse mode emission from the interior of the hollow cathode is strongest during the most negative part of the rf cycle at $78 \mathrm{~ns}$. This behaviour is typical of a capacitive discharge in the $\gamma$ mode and is attributed to the presence of ion induced secondary electron emission. ${ }^{15}$ Secondary electron yield from ion bombardment is proportional to incident ion energy, so a higher sheath voltage produces more secondary electrons. ${ }^{16}$ Additionally, the electrons ejected from the wall are accelerated back into the bulk plasma by the sheath. During the negative part of the rf cycle, the voltage on the powered electrode induces a sheath of up to several hundred volts. The expected abundance of high energy secondary electrons produced during the negative half-cycle can explain the increase in ionisation and excitation structure observed.

The propagation of plasma into the expansion chamber during the diffuse mode occurs when the applied voltage is positive, beginning at $20 \mathrm{~ns}$. Simulation of the Pocket Rocket system in argon shows that secondary electrons are emitted from the earthed electrodes during this part of the cycle. ${ }^{17}$ This is explained by considering that the plasma potential is maximised when the applied voltage is most positive. Since the grounded electrodes remain at $0 \mathrm{~V}$, the sheath voltage at the grounded surfaces is maximised during the time the voltage is positive. This also produces a burst of ion induced secondary electrons at the surface of the grounded electrode that faces the chamber. Therefore, it is suggested that these electrons are responsible for the timing of the extrusion of plasma during the diffuse mode.

In contrast to the diffuse mode, emission in the bright mode (shown as open circles in Figure 2) is peaked when the rate of change of voltage on the electrode is high. Figure 2(d) shows that inside the hollow cathode emission is strongest around 20 and $56 \mathrm{~ns}$. Furthermore, the production of secondary electrons around $78 \mathrm{~ns}$ is no longer visible, with emission minimised when the voltage is at its negative peak. Instead, the emission structure implies that the electrons are being heated through their interaction with the rf electric field. Along with inductive coupling, this behaviour also shares some similarities with field reversal effects in a capacitive discharge, as discussed by Vender, Czarnetzki, and others. ${ }^{18,19}$ However, the emission intensity of the bright mode makes it unlikely that field reversal effects are responsible for the mode transition. ${ }^{20}$ The sharp increase in power deposition efficiency seen in Figure 3 is consistent with the idea of a E-H transition occurring. However, it is important to note that there is no measurable hysteresis between the two modes, which is typically expected of the E-H mode transition. Furthermore, the system resistance is observed to increase from $5 \Omega$ to above $20 \Omega$. Typically, this is associated with an increase in plasma density. ${ }^{21}$ While the resistance of the plasma is seen to increase, its reactance goes down, suggesting a decrease in size of the sheaths as the plasma becomes less capacitive.

The vertical dark region approximately $3 \mathrm{~cm}$ to the left of the hollow cathode exit, seen in Figure 2(b) is visible once per rf cycle, between 56 and 80 ns. It can be observed as electrons propagate down the expansion tube causing ionisation and excitation of the neutral gas. The dark region is thought to be associated with the formation of a static supersonic shock in the expanding plasma. It is proposed that emission is suppressed in this region due to the depletion of plasma and neutral species density in the so called "zone of silence." 22 This depletion increases the mean free path for excitation and ionisation events in the supersonic region. For the shock to form, the gas in the expansion must exceed the sound speed. This can reasonably be attributed to the increase in translational temperature measured by the Fulcher $\alpha$ spectroscopy in the bright mode. The increase in temperature is primarily caused by the increased power deposition efficiency, leading to higher plasma density.

These measurements are consistent with an E-H mode transition in the plasma and suggest that the diffuse mode is primarily operating as a capacitive discharge. The increase in optical emission intensity and translation temperature of the gas after the mode transition both point to a significant increase in plasma density. The reduced voltage on the powered electrode during operation in the bright mode, combined with a higher power deposition efficiency corroborates this conclusion. Finally, the dominance of emission when the sheath voltage is varying most rapidly inside the hollow cathode is indicative of a sheath-electron interaction dominated heating mechanism.

In summary, time resolved measurements have been used to study the formation of two distinct modes in a low pressure, hydrogen hollow cathode discharge. The low brightness, diffuse mode, dominated by ion induced secondary electron emission, and the bright mode, which is apparently dominated by field-particle interactions. The diffuse mode is most likely a capacitive discharge operating in the $\gamma$ mode, while the power coupling of the bright mode is more uncertain. The change is possibly the result of an E-H mode transition, but displays very little hysteresis. Measurements 
of the electric fields within the hollow cathode discharge might provide further insight.

This research was partially funded by the Australian Research Council Discovery Project (DP1096653). The authors would like to express their gratitude to Andor Technologies for loaning the ICCD camera.

${ }^{1}$ L. Bardoš, Surf. Coat. Technol. 86, 648 (1996).

${ }^{2}$ W. A. Lanford and M. J. Rand, J. Appl. Phys. 49, 2473 (1978).

${ }^{3}$ R. M. Sankaran and K. P. Giapis, J. Appl. Phys. 92, 2406 (2002).

${ }^{4}$ W. M. M. Kessels, R. J. Severens, A. H. M. Smets, B. A. Korevaar, G. J. Adriaenssens, D. C. Schram, and M. C. M. van de Sanden, J. Appl. Phys. 89, 2404 (2001).

${ }^{5}$ C. Charles and R. Boswell, Plasma Sources Sci. Technol. 21, 022002 (2012).

${ }^{6}$ S. Dixon, C. Charles, R. Boswell, W. Cox, J. Holland, and R. Gottscho, J. Phys. D: Appl. Phys. 46, 145204 (2013).

${ }^{7}$ N. Mericam-Bourdet, M. Laroussi, A. Begum, and E. Karakas, J. Phys. D: Appl. Phys. 42, 055207 (2009).

${ }^{8}$ J. Schulze, T. Gans, D. O'Connell, U. Czarnetzki, A. R. Ellingboe, and M. M. Turner, J. Phys. D: Appl. Phys. 40, 7008 (2007).

${ }^{9}$ T. Gans, V. S. von der Gathen, and H. F. Döbele, Plasma Sources Sci. Technol. 10, 17 (2001).
${ }^{10}$ M. Teschke, J. Kedzierski, E. Finantu-Dinu, D. Korzec, and J. Engemann, IEEE Trans. Plasma Sci. 33, 310 (2005).

${ }^{11}$ A. Greig, C. Charles, R. Hawkins, and R. Boswell, Appl. Phys. Lett. 103, 074101 (2013).

${ }^{12}$ C. Charles, J. Dedrick, R. W. Boswell, D. Connell, and T. Gans, Appl. Phys. Lett. 103, 124103 (2013).

${ }^{13}$ S. Astashkevich, M. Käning, E. Käning, N. Kokina, B. Lavrov, A. Ohl, and J. Röpcke, J. Quant. Spectrosc. Radiat. Transfer 56, 725 (1996).

${ }^{14}$ M. Abdel-Rahman, T. Gans, V. S. von der Gathen, and H. F. Döbele, Plasma Sources Sci. Technol. 14, 51 (2005).

${ }^{15}$ S. Y. Moon, J. K. Rhee, D. B. Kim, and W. Choe, Phys. Plasmas 13, 033502 (2006).

${ }^{16}$ A. V. Phelps and Z. L. Petrovic, Plasma Sources Sci. Technol. 8, R21 (1999).

${ }^{17}$ A. Greig, C. Charles, and R. W. Boswell, private communication (2014).

${ }^{18}$ D. Vender and R. Boswell, IEEE Trans. Plasma Sci. 18, 725 (1990).

${ }^{19}$ U. Czarnetzki, D. Luggenhölscher, and H. F. Döbele, Plasma Sources Sci. Technol. 8, 230 (1999).

${ }^{20}$ M. Abdel-Rahman, V. S. von der Gathen, and T. Gans, J. Phys. D: Appl. Phys. 40, 1678 (2007).

${ }^{21}$ R. B. Piejak, V. A. Godyak, and B. M. Alexandrovich, Plasma Sources Sci. Technol. 1, 179 (1992).

${ }^{22}$ P. Vankan, S. Mazouffre, R. Engeln, and D. C. Schram, Phys. Plasmas 12, 102303 (2005). 